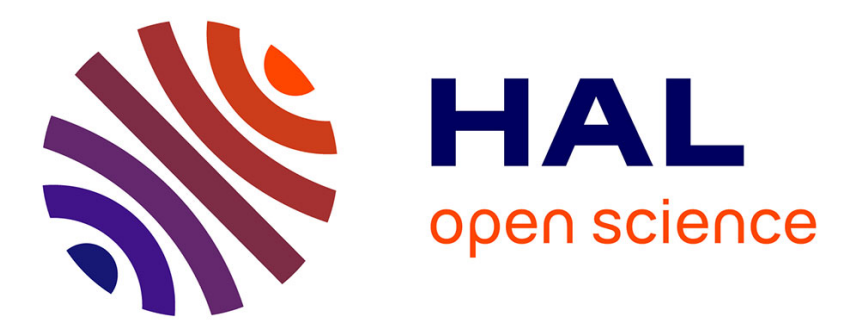

\title{
Energy balance and mixing between waves and eddies in stably stratified turbulence
}

\author{
H. Lam, A. Delache, F S Godeferd
}

\section{To cite this version:}

H. Lam, A. Delache, F S Godeferd. Energy balance and mixing between waves and eddies in stably stratified turbulence. Journal of Fluid Mechanics, 2021, 923, 10.1017/jfm.2021.589 . hal-03397106v2

\section{HAL Id: hal-03397106 \\ https://hal.science/hal-03397106v2}

Submitted on 15 Nov 2021

HAL is a multi-disciplinary open access archive for the deposit and dissemination of scientific research documents, whether they are published or not. The documents may come from teaching and research institutions in France or abroad, or from public or private research centers.
L'archive ouverte pluridisciplinaire HAL, est destinée au dépôt et à la diffusion de documents scientifiques de niveau recherche, publiés ou non, émanant des établissements d'enseignement et de recherche français ou étrangers, des laboratoires publics ou privés. 


\title{
Energy balance and mixing between waves and eddies in stably stratified turbulence
}

\author{
H. $\operatorname{Lam}^{1} \dagger$, A. Delache ${ }^{1,2}$ and F. S. Godeferd ${ }^{1}$ \\ ${ }^{1}$ Laboratoire de Mécanique des Fluides et d'Acoustique, Univ Lyon, École centrale de Lyon, \\ INSA Lyon, Université Claude Bernard Lyon I, CNRS, France \\ ${ }^{2}$ Laboratoire de Mécanique des Fluides et d'Acoustique, Univ Lyon, site de Saint-Étienne, \\ Université Jean Monnet de Saint-Étienne, CNRS, France
}

(Received $\mathrm{xx}$; revised $\mathrm{xx}$; accepted $\mathrm{xx}$ )

We explore the strong stratification regime of stably stratified turbulence and the intermediate regime towards the viscosity-affected stratified state. 3D velocitydensity fields from Direct Numerical Simulations are decomposed into Internal Gravity Waves (IGW) and eddy motion based on Riley's decomposition (Riley et al. 1981) extended to account for the space-time properties of waves, their modification by vertically-sheared horizontal flow and the vertical mixing by eddies (Lam et al. 2020). We establish the evolution equations for the IGW and eddy parts separately. Up to buoyancy Reynolds number $R e_{b} \sim 1$, we observe a large exchange of energy that pumps energy from IGW to eddy. For $R e_{b}>1$, IGW and eddy dynamics seem to be separate and no global exchange is observed. Our decomposition permits to compute the contributions to the mixing coefficient in terms of IGW and eddy. At the largest $R e_{b}$ considered, the mixing due to eddies is four times the mixing due to waves.

Key words: In line

\section{Introduction}

In stably stratified turbulence, Internal Gravity Waves (IGW) and eddies are closely entangled and interact with each other at different scales, as observed in the ocean (see e.g. Cusack et al. 2020). Many studies focus on different kinds of interactions, separately. First, the wave-vortex interaction concerns the propagation of IGW through large quasi-geostrophic eddy flow (Müller 1976) during which energy is transfered from eddy to waves. The eddies thus appear to deviate rays of IGW (Moulin \& Flór 2006). Second, the wave-wave interaction was examined starting from the isolated triadic point of view (Olbers 1976), then considering a stochastic field composed of many resonant triadic interactions (Müller et al. 1986) and finally extended to the wave turbulence formalism (Lvov et al. 2010). The creation of IGWs in a surrounding quiescent region due to a localized stratified turbulent cloud has been studied by Maffioli et al. (2014). Lelong \& Riley (1991) studied the weakly non-linear interactions in a highly stratified system between a vortical mode (i.e. a horizontal rotating eddy) and

$\dagger$ Email address for correspondence: henri.lam@ec-lyon.fr 
an IGW. They show that the vortical mode acts as a catalyst and facilitates the energy transfer between waves.

Nevertheless, in stably stratified turbulence, mixing by waves and eddies occurs over a wide range of scales, rendering difficult their separation and the precise identification of mutual interactions. According to Brethouwer et al. (2007), several regimes of stratified turbulence are found depending on the Froude number $F r=\varepsilon_{u} / N u_{h}^{2}$ and the buoyancy Reynolds number $R e_{b}=\varepsilon_{u} / \nu N^{2}$ where $\varepsilon_{u}$ is the kinetic energy dissipation, $u_{h}$ is the rms horizontal velocity, $N$ the Brunt-Väisälä frequency and $\nu$ the viscosity. For a strong stratification, at $F r \ll 1$ and $R e_{b} \ll 1$, the regime is a viscosity-affected stratified flow (VASF) and the flow is dominated by large smooth and stable horizontal layers and few turbulent-like structures, such as vortex tubes, are observed. This flow appears to be characteristic of a large-scale vortical mode (Waite \& Bartello 2004). At $F r \ll 1$ and $R e_{b} \gg 1$, the regime is strongly stratified turbulence (SST) where large vertically sheared horizontal flow (VSHF) and three-dimensional (3D) overturning structures are observed. In order to separate a turbulent field into eddy and wave parts, Riley et al. (1981) first proposed a 3D spatial decomposition. This decomposition was extensively used for stably stratified flow with or without rotation in many theoretical and numerical studies that explored different properties of IGW, eddies, VSHF and their interactions in terms of energy, transfer and scale dependence (see e.g. Godeferd \& Cambon (1994), Bartello (1995), Smith \& Waleffe (2002), Kimura \& Herring (2012), Herbert et al. (2016)). This approach appears to be relevant at small Froude number $\mathrm{Fr} \ll 1$ and low buoyancy Reynolds number $R e_{b} \ll 1$ where the eddies are mostly horizontal and the vertical motion and density field are associated to IGW (Lelong \& Riley 1991). Nevertheless, when $R e_{b}$ increases, as in the SST regime, parts of the vertical velocity and density fields are linked to vertical mixing and therefore not to waves. Moreover, IGW are characterized by their dispersion relation $\omega_{r}(\mathbf{k})=N \cos \theta(\mathbf{k})$ where $\theta(\mathbf{k})$ is the angle of the wave-vector $\mathbf{k}$ with the horizontal plane. Clearly, Riley's decomposition is a spatial decomposition and does not reflect the temporal properties of the waves, since it includes all frequencies of the flow motion, even outside the dispersion relation. Therefore, the dispersion relation of IGW cannot be characterized with this decomposition.

Alternative approaches have been developed, for example by selecting only a few Fourier modes (Lindborg \& Brethouwer 2007), and detecting in their temporal signal the presence of frequency peaks linked to their wave vector by the dispersion relation, which is a signature of IGW. Recent detailed analyses have been proposed to study waves in turbulence: in stratified turbulence, a global signature of IGW was observed in experiments of Savaro et al. $(2020)$ and in numerical simulations by Di Leoni \& Mininni (2015), and Maffioli et al. (2020) clearly characterized the presence of IGW by using a temporal analysis of reduced energy from Riley's decomposition.

However, there is a heavy computational cost to a complete wave/eddy separation, so that simplifying assumptions are used in the above-mentioned methods: horizontal isotropy, and the fact that transport of IGW occurs in a homogeneous distribution of VSHF. The latter assumption discards possible variations in time and space of the transporting motion that in principle modifies significantly the waves dispersion relation. Recently, Lam et al. $(2020)$ extended Riley's decomposition by taking into account the 3D spatial and temporal properties of fields. This method permits to extract the 3D fields of IGW and eddies separately, 
accounting for the overturning of density and vertical velocity. It is based on a joint analysis in space and time by performing the full $4 \mathrm{D}$ Fourier transform. This is computationally expensive and requires adequate numerical resolution in time and in space.

In the present work, we use a more advanced and versatile version of this method which also takes into account the spatial and temporal variation of the VSHF on the dispersion relation. Nevertheless, in order to achieve statistical stationarity of the flow, the VSHF is damped in our DNS but it plays a significant role even if it does not dominate the overall structure of the flow. It is then possible to obtain a precise budget of wave and eddy energies, their interactions and the different fluxes. Thanks to this budget, it becomes possible to clearly decompose the mixing coefficient $\Gamma$ used in oceanography to model vertical turbulent mixing (Mashayek et al. 2017; Ivey et al. 2008), into a part coming from the waves and another part coming from the eddies. In order to improve modelling, it is possible to analyse the mixing done by eddies due mostly to the breaking of IGW (MacKinnon 2017) and the mixing by IGW themselves.

In the following, we thus first present in section 2 the method used for separating waves and eddies in stratified turbulence, in order to apply it to fields coming from DNS presented in section 3. The energetics is analyzed in section 4 . and consequences on mixing are discussed in section 5 . Conclusions are drawn in section 6 .

\section{Extraction of eddies and waves in stratified turbulence}

\subsection{Governing equation}

We consider a linearly stratified incompressible fluid, and the corresponding Navier-Stokes equation under the Boussinesq approximation:

$$
\begin{array}{r}
\partial_{t} \mathbf{u}+\boldsymbol{\omega} \times \mathbf{u}+\nabla p-\nu \nabla^{2} \mathbf{u}=b \mathbf{z}+\mathbf{F}_{\mathbf{u}} \\
\partial_{t} b+\mathbf{u} \cdot \nabla b-\mathcal{X} \nabla^{2} b=-N^{2} u_{z} .
\end{array}
$$

The velocity vector is $\mathbf{u}=\left(u_{x}, u_{y}, u_{z}\right)$ with vorticity $\boldsymbol{\omega}=\nabla \times \mathbf{u}$, and $p$ is the modified pressure field. The kinematic viscosity is $\nu$ and the thermal diffusivity $\mathcal{X}$. The Brunt-Väisälä frequency is $N$ so that the buoyancy field $b$ is the negative fluctuation of density around the mean constant gradient $N^{2}$. $\mathbf{z}$ is the unit vector of polar direction, also vertical. The flow can be set in motion via the body force $\mathbf{F}_{\mathbf{u}}$. All equations and parameters are dimensionless by reference to length and scale.

Our method for extracting the IGW from the full flow is based on the characteristics of IGW. Indeed, classical IGW are plane-wave solutions of the inviscid equations, obtained by removing the non-linear terms of equations (2.1)- 2.2), and identifying their dispersion relation $\omega_{r}(\mathbf{k})$. However, in the presence of largescale advection (such as the VSHF), this dispersion relation $\omega_{r}(\mathbf{k})$ is modified. If one takes only into account the sweeping effect by large structures on IGW, assimilated to an advecting velocity field $\mathbf{c}$, the modification of IGW characteristics can be modelled by the following linearized equations:

$$
\begin{gathered}
\partial_{t} \mathbf{u}_{G}+\mathbf{c} \cdot \nabla \mathbf{u}_{G}+\nabla p_{G}-\nu_{G} \nabla^{2} \mathbf{u}_{G}=b_{G} \mathbf{z} \\
\partial_{t} b_{G}+\mathbf{c} \cdot \nabla b_{G}-\mathcal{X}_{G} \nabla^{2} b_{G}=-N^{2} u_{z, G}+F_{b}
\end{gathered}
$$

and we shall use density forcing via $F_{b}$ with new variables named $\mathbf{u}_{G}, b_{G}, \nu_{G}$, 
$\mathcal{X}_{G}$ associated to equation (2.3). In our modelling of the modification of IGW's characteristics, we do not take into account the refraction of waves by the vertical shear $\left(i . e . u_{z} \partial_{z} c\right)$ as it does not change significantly our results: our DNS show that $\partial_{z} c \sim 0.04 \ll N$, and the corresponding analytical evaluation and preliminary numerical tests show that the dispersion relation is not significantly modified in that case with respect to the sweeping effect.

The characteristics of the IGW are obtained by solving equations $(2.3)$ and are used to extract the wave part from the full velocity obtained by equation (2.2). In both cases, it is useful to use the same basis of description for equation (2.2) and (2.3). In Fourier space, since incompressibility imposes that the velocity vector be perpendicular to the associated wave vector, we introduce polar-spherical coordinates where the unit vectors of the so-called Craya-Herring frame are $\left(\mathbf{e}^{t}, \mathbf{e}^{p}, \mathbf{e}^{k}\right)$ defined with respect to the vertical axis $\mathbf{z}$ :

$$
\mathbf{e}^{t}=\frac{\mathbf{k} \times \mathbf{z}}{|\mathbf{k} \times \mathbf{z}|}, \quad \mathbf{e}^{p}=\frac{\mathbf{k} \times(\mathbf{k} \times \mathbf{z})}{|\mathbf{k} \times(\mathbf{k} \times \mathbf{z})|}, \quad \mathbf{e}^{k}=\mathbf{k} / k .
$$

Therefore, in this basis, the Fourier-transformed velocity writes $\hat{\mathbf{u}}(\mathbf{k}, t)=$ $\hat{u}^{t}(\mathbf{k}, t) \mathbf{e}^{t}+\hat{u}^{p}(\mathbf{k}, t) \mathbf{e}^{p}+\hat{\mathbf{u}}^{s}\left(k_{z}, t\right)$ where ${ }^{\wedge}$ represents the Fourier transform in space (details in Lam et al. (2020)). The toroidal part $\hat{u}^{t}$ is horizontal and corresponds to a horizontal wave vector $k_{h} \neq 0$ with $k_{h}=\left(k_{x}^{2}+k_{y}^{2}\right)^{1 / 2}$, and the poloidal part $\hat{u}^{p}$ contains both horizontal and vertical velocity and thus corresponds to IGW motion. In physical space, the corresponding velocity fields are $u^{t}$ and $u^{p}$. This decomposition excludes the VSHF mode - noted in Fourier space $\hat{\mathbf{u}}^{s}\left(k_{z}, t\right)=\hat{\mathbf{u}}\left(k_{h}=0, k_{z}, t\right)$ - which corresponds to the horizontal velocity field at exactly vertical wave vector such that $k_{h}=0$. This results from the mathematical definition of polar-spherical coordinates based on an axis of symmetry borne by $\mathbf{z}$.

\subsection{Method of extraction}

In the original decomposition by Riley et al. (1981), the Craya-Herring decomposition is applied on velocity fields of stratified turbulence in the limit of small Froude number. The poloidal part $u^{p}$ is clearly the velocity of IGW and the toroidal part $u^{t}$ is the non-wave part which coincides with a two-dimensional horizontally rotational velocity associated to eddy dynamics, and often called 'vortical mode'. This decomposition appears to be relevant at low buoyancy Reynolds number $R e_{b} \ll 1$ and at low Froude number $F r \ll 1$ where the flow consists of an interaction between vortical modes at large scale and IGW (see Lelong \& Riley (1991)). At larger $R e_{b}$, a part of vertical velocity $u^{p}$ and of density $b$ is linked to vertical mixing, and therefore not to waves. To account for this, Lam et al. (2020) extended Riley's decomposition to explicitly include the spacetime properties of waves and their transport by a constant advecting velocity $\mathbf{c}$ estimated from the energy of the VSHF.

In the case of a constant advection velocity c, homogeneous in time and space, Riley's decomposition applied to equation (2.3) is rigorous. Indeed, an analytical solution of Green's function of the IGW can be obtained from the linearized equations (2.3) forced by Dirac functions in time and space $F_{b}=\delta(t) \delta(x)$. Solved in the four-dimensional Fourier domain in space and time $(\mathbf{k}, \omega)$, and denoted , the Green's function of IGWs is composed only by the poloidal part denoted $\tilde{u}_{G, a}^{p}$ 
and by the density denoted $\tilde{b}_{G, a}$ :

$$
\begin{aligned}
& \tilde{b}_{G, a}(\mathbf{k}, \omega)=\frac{1}{2}\left\{\left[\mathcal{X} k^{2}+\mathrm{i}\left(\omega+\omega_{c}^{+}(\mathbf{k})\right)\right]^{-1}+\left[\mathcal{X} k^{2}+\mathrm{i}\left(\omega-\omega_{c}^{-}(\mathbf{k})\right)\right]^{-1}\right\} \\
& \tilde{u}_{G, a}^{p}(\mathbf{k}, \omega)=\frac{\mathrm{i}}{2 N}\left\{\left[\nu k^{2}+\mathrm{i}\left(\omega+\omega_{c}^{+}(\mathbf{k})\right)\right]^{-1}-\left[\nu k^{2}+\mathrm{i}\left(\omega-\omega_{c}^{-}(\mathbf{k})\right)\right]^{-1}\right\}
\end{aligned}
$$

where the analytical dispersion relation $\omega_{c}^{ \pm}(\mathbf{k})=\mathbf{c} \cdot \mathbf{k} \pm \omega_{r}(\mathbf{k})$ is $\omega_{r}$ modified to include $\mathbf{c} \cdot \mathbf{k}$ that represents the sweeping effect, similar to Doppler shift. The toroidal part $\tilde{u}_{G, a}^{t}(\mathbf{k}, \omega)$ is constant in time, i.e. with a zero frequency, and it is the vortical mode which is the rotational horizontal flow i.e. $k_{h} \neq 0$ (see note 14 in Smith \& Waleffe (1999)). Our new technique is based on a filter $\zeta(\mathbf{k}, \omega)$ in four-dimensional space $(\mathbf{k}, \omega)$ applied on the density field $\tilde{b}$ and velocity field $\tilde{\mathbf{u}}$ from DNS. The wave part is obtained from the $\tilde{b}$ and $\tilde{\mathbf{u}}$ fields and by filtering the frequencies $\omega$ associated with each wave vector $\mathbf{k}$ for the velocity component $\tilde{u}^{p}$ and density component $\tilde{b}$. In Lam et al. (2020), this filter is based on the analytical dispersion relation: $\zeta(\mathbf{k}, \omega)=1$ when $\omega=\omega_{c}^{ \pm}(\mathbf{k})$ where $\mathbf{c}$ is horizontal and its amplitude $c$ varies between $\left[-c_{V}, c_{V}\right], c_{V}$ being the rms of VSHF amplitude. This supposes that the large-scale such VSHF is modelled as a homogeneous flow that transports velocity fluctuations. In practice, the velocity of VSHF varies along the $z$ axis with time i.e. $\mathbf{c}(z, t)$ and it is not possible to derive an analytical solution such as equation (2.5). More generally, no theoretical dispersion relation can easily be found when $\mathbf{c}$ is inhomogeneous. To overcome this difficulty we simulate numerically the Green's function.

For an inhomogeneous advecting velocity $\mathbf{c}(z, t)$, we generalize a property that appears in the analytical wave solution (2.5): when the frequency $\omega \rightarrow \omega_{c}^{ \pm}(\mathbf{k})$, the density energy $\left|\tilde{b}_{G}\right|^{2}$ peaks, only damped by viscosity. This permits to compute the Green's function relevant for the linearized equations (2.3) where $\mathbf{c}(z, t)$ is the inhomogeneous VSHF, numerically extracted from DNS i.e. $\hat{\mathbf{c}}\left(k_{z}, t\right)=$ $\hat{\mathbf{u}}\left(k_{h}=0, k_{z}, t\right)$ and $F_{b}=\sum_{\mathbf{x}, t} \delta(\mathbf{x}) \delta(t)$ is an inhomogeneous distribution of Dirac functions in space and time. The extended filter is obtained simply by computing the energy of $\left|\tilde{b}_{G}\right|^{2}$ with respect to the space variable $\mathbf{k}$ and time variable $\omega$. When the energy $\tilde{b}_{G}$ peaks, for $\left(\mathbf{k}_{0}, \omega_{0}\right)$, one defines the corresponding spectral region as characteristic of a wave, so we set $\zeta\left(\mathbf{k}_{0}, \omega_{0}\right)=1$. In practice, to reach the peak, the extended filter is therefore defined as:

$$
\text { if }\left|\tilde{b}_{G}(\mathbf{k}, \omega)\right|^{2} \geqslant \beta^{-1} \max _{\omega}\left|\tilde{b}_{G}(\mathbf{k}, \omega)\right|^{2} \text { then } \zeta(\mathbf{k}, \omega)=1 \text { else } \zeta(\mathbf{k}, \omega)=0
$$

where the real parameter $\beta=100$ is selected in order to capture all peaks of energy on a range of frequencies and take into account not only the spread of peak over frequencies due to diffusivity but inaccuracy due to spatial and temporal discretization (see section 3). The filter $\zeta(\mathbf{k}, \omega)$ implicitly defines a new dispersion relation $\omega_{G}(\mathbf{k})$ that accounts for the inhomogeneity of $\mathbf{c}(z, t)$. It can be applied on the DNS fields to separate wave part $(\zeta=1$, label ' $w$ ') from eddy part $(\zeta=0$, label ' $e$ '):

$$
\begin{gathered}
\tilde{\mathbf{u}}^{w}(\mathbf{k}, \omega)=\zeta(\mathbf{k}, \omega) \tilde{u}^{p}(\mathbf{k}, \omega) \mathbf{e}^{p} \\
\tilde{\mathbf{u}}^{e}(\mathbf{k}, \omega)=\tilde{u}^{t}(\mathbf{k}, \omega) \mathbf{e}^{t}+(1-\zeta(\mathbf{k}, \omega)) \tilde{u}^{p}(\mathbf{k}, \omega) \mathbf{e}^{p} \\
\tilde{b}^{w}(\mathbf{k}, \omega)=\zeta(\mathbf{k}, \omega) \tilde{b}(\mathbf{k}, \omega) \text { and } \tilde{b}^{e}(\mathbf{k}, \omega)=(1-\zeta(\mathbf{k}, \omega)) \tilde{b}(\mathbf{k}, \omega) .
\end{gathered}
$$

In this decomposition IGW are defined as being the poloidal and buoyancy 
components close to the dispersion relation. Eddies are defined here as elements that are not a wave as IGW and that are not the VSHF either. In this article, we employ the term 'eddy part' for convenience, admitting that it is initially defined by what it is not. Nevertheless, the eddy part mostly contains large-scale vortical modes, which are exactly toroidal components, but also small-scale threedimensional structures which are a mixture of poloidal and toroidal components.

The decomposition of $\tilde{\mathbf{u}}^{w}$ agrees with the observations of Maffioli et al. (2020) that only the poloidal components recover the waves dispersion relation. The wave part in equations (2.7) contains only frequencies corresponding to the dispersion relation and the eddy part contains a part of vertical velocity and the density. Applying the inverse four-dimensional Fourier transform from frequency space $(\mathbf{k}, \omega)$ to physical space $(\mathbf{x}, t)$ yields $\mathbf{u}^{a}(\mathbf{x}, t)=\sum_{\mathbf{k}, \omega} \tilde{\mathbf{u}}^{a}(\mathbf{k}, \omega) e^{-\mathrm{i} \mathbf{k} \cdot \mathbf{x}-\mathrm{i} \omega t}, b^{a}(\mathbf{x}, t)=$ $\sum_{\mathbf{k}, \omega} \tilde{b}^{a}(\mathbf{k}, \omega) e^{-\mathrm{i} \mathbf{k} \cdot \mathbf{x}-\mathrm{i} \omega t}$ ( $a$ stands for $w, e$ and remaining part $s$ ), and one obtains the decomposition of velocity and density:

$$
\mathbf{u}(\mathbf{x}, t)=\mathbf{u}^{w}(\mathbf{x}, t)+\mathbf{u}^{e}(\mathbf{x}, t)+\mathbf{u}^{s}(\mathbf{x}, t) \text { and } b(\mathbf{x}, t)=b^{w}(\mathbf{x}, t)+b^{e}(\mathbf{x}, t)
$$

where the VSHF has been identified as a mean flow in physical space $\mathbf{u}^{s}(\mathbf{x}, t)$ coming from its definition in Fourier space as $\hat{\mathbf{u}}^{s}(\mathbf{k}, t)=\hat{\mathbf{u}}\left(k_{h}=0, k_{z}, t\right)$.

This decomposition permits to define an orthogonal basis and an inner product in vector function space, by using the complete set of unit vector functions $e^{\mathbf{i k} \cdot \mathbf{x}}$ and $e^{\mathrm{i} \omega t}$. For two functions $\hat{f}$ and $\hat{g}$, we thus define an inner product in terms of wavevector $\mathbf{k}$ and time $t$, as $\left[\hat{f}(\mathbf{k}, t), \hat{g}\left(\mathbf{k}^{\prime}, t\right)\right] \equiv \frac{1}{T} \int_{T} \hat{f}(\mathbf{k}, t) \overline{\hat{g}\left(\mathbf{k}^{\prime}, t\right)} \delta_{\mathbf{k}-\mathbf{k}^{\prime}} \mathrm{d} t$ where $T$ is the considered time span and ${ }^{-}$is the complex conjugate. Due to the orthogonality of vector space functions and orthogonality of Fourier velocity with wavevector space $\mathbf{k}$ from incompressibility, one shows the orthogonality between wave, eddy, and shear parts: $\left[\hat{b}^{i}(\mathbf{k}, t), \hat{b}^{j}\left(\mathbf{k}^{\prime}, t\right)\right] \neq 0$ and $\left[\hat{u}_{m}^{i}(\mathbf{k}, t), \hat{u}_{n}^{j}\left(\mathbf{k}^{\prime}, t\right)\right] \neq 0$ only if $i=j$ and $\mathbf{k}=\mathbf{k}^{\prime}$ (where $i, j$ stand for $w, e$ or $s$, and $m, n$ stand for space direction $x, y$, or $z)$. Moreover, the overall energetic content is $<\hat{f}, \hat{g}>=\sum_{\mathbf{k}} \Re[\hat{f}(\mathbf{k}, t), \hat{g}(\mathbf{k}, t)]$.

\section{Numerical methodology and parameters}

\subsection{Parameters space}

In a first kind of DNS we run, equations (2.1) and 2.2 are solved using a standard pseudo-spectral algorithm in a $2 \pi$-periodic three-dimensional spatial domain. A phase shifting method is used to treat aliasing in the non-linear term (see Lam et al. (2020) for details). The Prandtl number is $\operatorname{Pr}=\nu / \mathcal{X}=1$. Ten numerical simulations have been run with the parameters shown in table 1 at resolutions $256^{3}$ and $512^{3}$. The exploration of parameters is mainly based on $512^{3}$ points, the lower resolution of $256^{3}$ points is used to confirm and explore trends. We have plotted in Figure 1 the exploration points in the parameter space $\left(F r, R e_{b}\right)$, along with data from Maffioli et al. (2016) and Garanaik \& Venayagamoorthy (2019). According to Brethouwer et al. (2007), our parameters explore a VASF regime which contains weak IGW interactions where wave anisotropy extends to small scales $\left(F r \ll 1\right.$ and $\left.R e_{b} \ll 1\right)$, as well as a SST regime where the scale of wave anisotropy is distinct from small dissipative scales $\left(F r \ll 1\right.$ and $\left.R e_{b} \gg 1\right)$. The exploration of these two regimes also induce a modification of the Taylor- 
$512^{3}$ points with $\nu=1 / 700$

$\begin{array}{cccccccccccc}N & F r & R e_{b} & R e_{h} & R e_{\lambda} & D_{E} & S_{E}(\%) & \omega_{\max } & \omega_{\min } & u_{r \operatorname{ms}} k_{\max } & k_{\max } \eta & \begin{array}{c}\Delta t \\ \left(\times 10^{-4}\right)\end{array} \\ 20 & 0.035 & 11 & 9000 & 146 & 760 & 0.3 & 1570 & 3 & 800 & 1.12 & 2 \\ 30 & 0.023 & 5 & 9500 & 148 & 1080 & 0.4 & 1570 & 3 & 810 & 1.12 & 2 \\ 50 & 0.013 & 1.8 & 10700 & 180 & 980 & 0.4 & 1570 & 3 & 890 & 1.12 & 2 \\ 70 & 0.007 & 0.9 & 18000 & 225 & 820 & 0.3 & 1570 & 3 & 990 & 1.12 & 2 \\ 100 & 0.004 & 0.5 & 31000 & 290 & 730 & 0.3 & 1570 & 3 & 1110 & 1.12 & 2 \\ 200 & 0.0014 & 0.1 & 51000 & 430 & 22 & 0.08 & 3140 & 6 & 1300 & 1.13 & 1 \\ 600 & 0.00045 & 0.01 & 49000 & 510 & 11 & 0.005 & 3140 & 6 & 1370 & 1.19 & 1\end{array}$

$256^{3}$ points with $\nu=1 / 250$

$\begin{array}{cccccccccccc}N & F r & R e_{b} & R e_{h} & R e_{\lambda} & D_{E} & S_{E}(\%) & \omega_{\max } & \omega_{\min } & u_{r \operatorname{ms}} k_{\max } & k_{\max } \eta & \Delta t \\ & & & & & & & & & & & \\ \left(\times 10^{-4}\right) \\ 70 & 0.014 & 0.7 & 3600 & 94 & 2500 & 1 & 1570 & 3 & 423 & 1.18 & 4 \\ 200 & 0.0095 & 0.35 & 3900 & 109 & 420 & 0.16 & 1570 & 3 & 450 & 1.19 & 5 \\ & 0.0022 & 0.04 & 8300 & 175 & 74 & 0.03 & 1570 & 3 & 557 & 1.21 & 4\end{array}$

Table 1: List of parameters in the seven DNS runs. $R e_{h}=u_{h}^{4} /\left(\varepsilon_{u} \nu\right)$ is the horizontal Reynolds number, $S_{E}$ is the ratio of energy of shear flow over the total kinetic energy, $D_{E}$ is the ratio of density of shear energy per point against the total kinetic energy per point, $\omega_{\max }=\pi / \Delta t^{\prime}$ and $\omega_{\min }=2 \pi / T$ are the maximum and minimum pulsation solved.
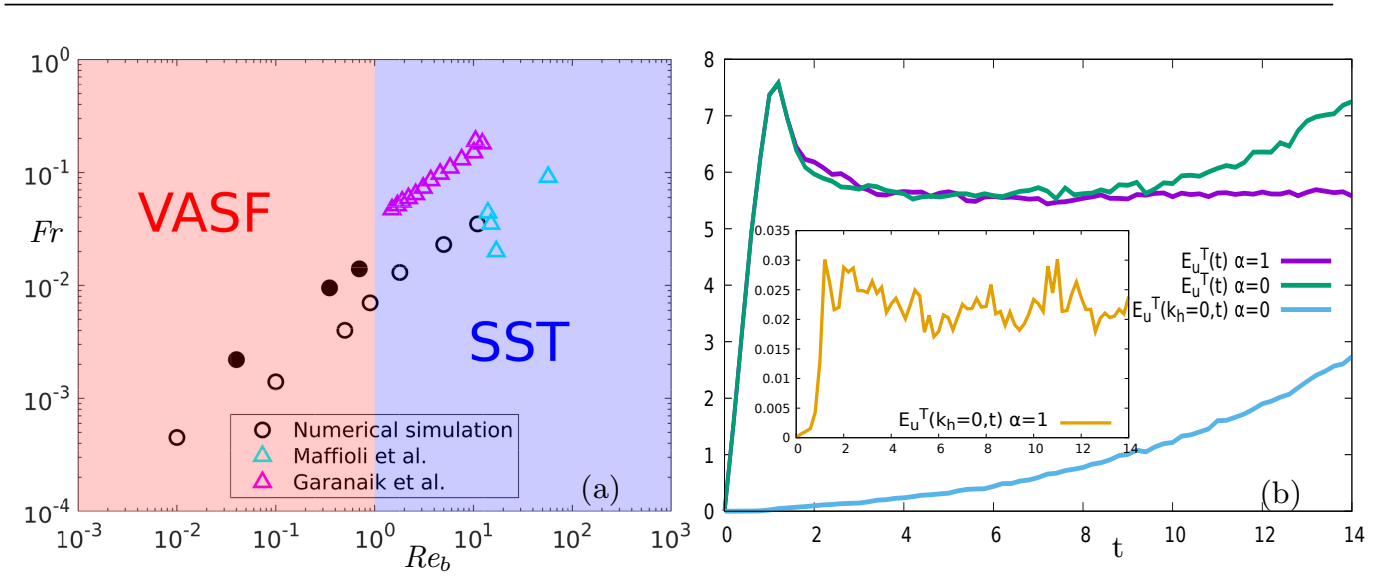

Figure 1: (a) Our parameters of the numerical simulations (open circle for $512^{3}$ points and filled circle for $256^{3}$ points). For reference, the DNS of Maffioli et al. (2016) (from $96^{3}$ points to more than $1024^{3}$ points) and Garanaik \&

Venayagamoorthy (2019) $\left(512^{3}\right.$ points) are shown. (b) Total kinetic energy $E_{u}^{T}(t)$ and VSHF energy $E_{u}\left(k_{h}=0, t\right)$ for $\alpha=1$ and $\alpha=0$ for $R_{b}=5$ and $\mathrm{Fr}=0.023$.

length-based Reynolds number $R e_{\lambda}=u_{r m s} \lambda / \nu$ with $\lambda$ the Taylor scale and $u_{\text {rms }}$ the rms velocity. The regimes studied in our numerical simulations and in other numerical simulations are shown in figure 1 a. The figure shows that the two resolutions $256^{3}$ and $512^{3}$ explore different regions of parameters space $\left(F r, R e_{b}\right)$ and we expect this to change the characteristics of the transition regime between the VASF and SST regime. Additional few points in parameter space at $256^{3}$ 
resolution permit to explore a slight variation of $R e_{b}$ and $F r$. The two parameters $F r$ and $R e_{b}$ are of course dependent on one another since $R e_{b}=F r^{2} R e_{h}$, via the horizontal Reynolds number $R e_{h}=u_{h}^{4} /\left(\varepsilon_{u} \nu\right)$ defined by Maffioli et al. (2016), that accounts for the horizontal turbulence intensity. The $256^{3}$ simulations have almost one order of magnitude lower $R e_{h}$ than $512^{3}$ simulations (see table 1 ) for similar $\left(R e_{b}, F r\right)$. By adjusting the resolution, one can therefore study the variation of the dynamical system either by setting $F r$ and weakly increasing $R e_{b}$ (from low to high resolution), or by setting $R e_{b}$ and weakly increasing $F r$ (from high to low resolution) in the parameter map.

\subsection{Numerical parameters}

The time step $\Delta t$ varies with the stratification $N$ to agree with the CFL condition. For the spatial resolution of $512^{3}$ points, the maximum wavenumber is $k_{\max }=241$ such that $k_{\max } \eta \sim 1.1, \eta$ being the Kolmogorov scale (see table 1). This moderate number of points is necessary because our wave/eddy decomposition requires many 3D fields in time. Turbulence reaches a statistically stationary state due to the added body force $F_{u}$ in equation (2.1), as in Maffioli et al. (2020) who injected a constant power $P=\int \mathbf{F}_{\mathbf{u}} \cdot \mathbf{u} \mathrm{d} v=10 . \mathbf{F}_{\mathbf{u}}$ is spectrally localized on a cylindrical spectral surface of horizontal wave number $k_{h}=4$ and vertical wave number $1 \leqslant k_{z} \leqslant 3$, away from the VSHF at $\hat{\mathbf{u}}\left(k_{h}=0, k_{z}\right)$. It forces the poloidal and toroidal parts of the velocity equally. Thus this choice allows on average the wave and vortex components of the flow (in the sense of Riley's decomposition) to be excited in equal proportions. The forced wavenumbers are at an angle $\theta_{f}$ between the wavevector $\mathbf{k}$ and the horizontal plane, in the range $0.72 \leqslant \theta_{f} \leqslant 1.31$, meaning that high frequencies close to $N$ are forced and a wave turbulence cascade may develop with lower frequency. To delay the emergence of VSHF at large scale, we add a friction term $\hat{\mathbf{F}}_{u}-\alpha \hat{\mathbf{u}}\left(\mathbf{k}_{h}=0, k_{z}\right.$ ) (with $\alpha=1$ ) as proposed by Le Reun et al. (2017) to stabilise the geostrophic mode in rotating turbulence. The latter authors also note that this term mimics the effect of a horizontal wall. It also helps the numerical simulation to reach a stationary state as shown in figure $1 \mathrm{~b}$. This figure shows the total kinetic energy $E_{u}^{T}(t)$ and the kinetic energy of VSHF $E_{u}^{T}\left(k_{h}=0, t\right)$ for $\alpha=1$ and $\alpha=0$. Both energies diverge when $\alpha=0$ but stay bounded when $\alpha=1$. This statistical stationarity allows to compute time Fourier transforms with less truncation-related spurious effects. Furthermore the divergence of the kinetic energy $E_{u}^{T}(t)$ for $\alpha=0$ is about the same as that of VSHF energy $E_{u}^{T}\left(k_{h}=0, t\right)$, meaning that roughly the same amount of energy is advected by the VSHF. The main difference between the two cases with and without friction is that the flow is significantly advected by VSHF in the first case $(\alpha=0)$ whereas this advection is much less in the case with friction $(\alpha=1)$.

Our simulations contrast with those of Maffioli et al. $(2020)$ in that we apply a friction term to quench VSHF to less than a few percent of the total kinetic energy, though still active enough to contribute to the flow structuration. We show in table 1 that the percentage of shear energy over the total kinetic energy $\left(S_{E}=E_{u}^{T}\left(k_{h}=0, t\right) / E_{u}^{T}(t)\right)$ is very low. However, we still consider the VSHF to be the main advecting flow.

We consider that all wavevectors $k_{x}, k_{y}, k_{z}\left(512^{3}\right.$ points $)$ are 'active' - i.e. they are prone to contributing significantly — in the total kinetic energy, whereas in the kinetic energy of the VSHF, we only consider as active wavevectors with $k_{h}=0$, at whatever $k_{z}$ (512 points). Then, from these total kinetic energy $E_{u}^{T}$ 
and kinetic energy of VSHF $E_{u}^{T}\left(k_{h}=0\right)$, it is possible to define the following average energy densities: $e_{K}=E_{u}^{T} / 512^{3}$ and $e_{\text {shear }}=E_{u}^{T}\left(k_{h}=0\right) / 512$. These average densities take explicitly into account the number of active wavenumbers they involve. Finally, we can define the average density ratio per wavevector by $D_{E}=e_{\text {shear }} / e_{K}$. The value $D_{E}$ is given in table 1 and shows the relative importance of the VSHF compared to the number of points in the DNS involved. $D_{E} \sim 1000$ shows that the VSHF importance per point is strong for weaker stratification, but decreases while still intense for higher stratification.

In a second kind of DNS we run in order to build the $\zeta$ function, in preparation for the DNS with $512^{3}$ points, the Green's function is simulated during $T=$ $10000 \Delta t$ by using equation (2.3) with the forcing term $F_{b}=\sum_{\mathbf{x}, t} \delta(\mathbf{x}) \delta(t)$ where each Dirac function is set at a random position and enforced at each time step $\Delta t$ during the first $100 \Delta t$. The initial condition of this calculation is zero. The velocity $\mathbf{c}$ comes from the $\operatorname{VSHF} \hat{\mathbf{u}}\left(k_{h}=0, k z\right)$ extracted every $\Delta t^{\prime}=10 \Delta t$ from the DNS, after it has reached statistical stationarity. To ensure that IGW are not dissipated, we use very small viscosity $\nu_{G}=\mathcal{X}_{G}=10^{-8}$ and we check that only the poloidal part $\hat{u}^{p}$ and the density $\hat{b}$ are active with respect to the toroidal part $\hat{u}^{t}$ that is close to machine-precision zero. We apply the FFT in time on 1000 fields of $\hat{b}_{G}$ extracted every $\Delta t^{\prime}$. For DNS with $256^{3}$ points the time step $\Delta t$ can be taken larger and result in a DNS with less iterations $(T=4000$ or $5000 \Delta t$ ) but with statistics written on the same time step $\Delta t^{\prime}=0.002$ as in numerical simulations with $512^{3}$ points. The time step is chosen very small in order to capture the sweeping effect from the full rms velocity $u_{r m s}$ on the highest frequency of eddies $u_{r m s} k_{\max }$, as validated in homogeneous and isotropic DNS simulation by Di Leoni et al. (2015). The highest frequency of eddies $u_{\mathrm{rms}} k_{\max }$ must be compared to maximum frequency $\omega_{\max }$ and minimum frequency $\omega_{\min }$ resolved by the numerical algorithm. The fields are not extracted at every $\Delta t$ both to reduce the memory cost and because in the DNS this time step comes mainly from the CFL constraint.

The value $\beta=100$ is based on the simulation of the Green's function under conditions similar to the analytical solution 2.5 for buoyancy $\tilde{b}_{G, a}$ which is a benchmark for our method. As explained after equation $(2.6), \beta$ is the cutoff parameter for identifying the spectral peaks. Two reasons render imprecise the capture of peaks in the simulation of Green's functions. First, even if very low (e.g. $\left.\nu_{G}=\mathcal{X}_{G}=10^{-8}\right)$, viscosity tends to smear the peaks around the resonance frequencies $\omega_{c}^{ \pm}$. Spectral discretization also adds to this smearing: for each wavevector $\mathbf{k}, 100 \%$ of energy is localized in a single frequency $\omega_{c}^{ \pm}(\mathbf{k})$ when analytically computed, whereas it is distributed over a bandwith of frequencies in simulations. The second reason is due to time discretization: the frequency $\omega_{c}^{ \pm}$is not exactly measured, but is approximated by the two closest discrete frequencies. These two mechanisms lead to a search for the set of points closest to the peak. When trying to capture the peak in a configuration similar to the analytical solution (??), we observe that the peaks span several orders of magnitude in amplitude over a bandwith of frequencies. In simulations, even if $100 \%$ of the energy is distributed over all frequencies, in practice a large percentage is still located in a small frequency range. In numerical simulations in the exact configuration of the analytical solution $\tilde{b}_{G, a}$, we adjusted $\beta$ to 100 because we observe that $95 \%$ of the total potential energy is selected as waves around a small bandwith of frequencies. This $95 \%$ value is retained for all the 

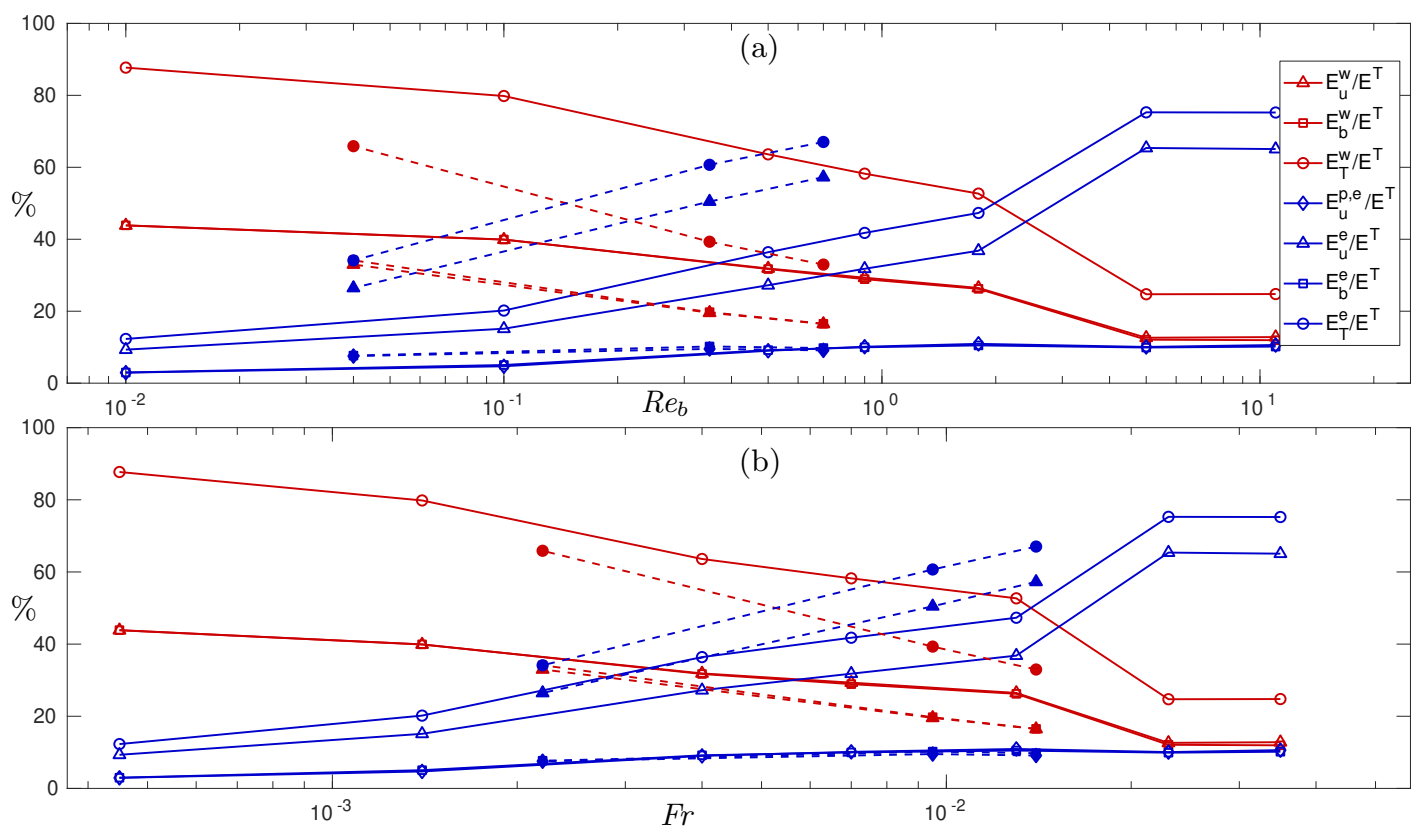

Figure 2: Evolution of the percentage of energy in waves and eddies

$\left(E_{i}^{e} / E^{T}, E_{i}^{w} / E^{T}\right)$ for kinetic, potential and total energy $(i=b, u, T$ respectively) against (a) $R e_{b}$; (b) Fr. Numerical simulations with $512^{3}$ points are shown with open symbols and solid lines, and numerical simulations with $256^{3}$ points are shown with filled symbols and dotted lines.

simulations of the Green's function from equation (2.3). Choosing lower $\beta$ means that less potential energy from equation $(2.3)$ would be considered as waves, meaning that some eddies would be assigned as waves. Conversely, choosing a higher $\beta$ would not change much in the wave energy in equation (2.3) and might increase the number of eddies associated to IGW. In configurations other than the analytical solution, simulations of Green's functions also show that more or less $95 \%$ of the total potential energy is preserved as waves.

Eventually, the $\zeta$ filter is applied to the $\hat{u}^{t}, \hat{u}^{p}, \hat{b}$ fields extracted every $\Delta t^{\prime}$ from the stationary part of the first type of DNS, i.e. over the same period $T$ of extraction of VSHF. The inverse FFT then yields the decomposition (2.8). No Hann window is used to avoid modifying the signal and energy, except for the creation of $\zeta$. All following statistics are averaged over $T_{0}=600 \Delta t^{\prime}$ in the middle of the time domain $T$. They do not change significantly if $T_{0}$ is halved, ensuring they are converged. All frequencies are adequately resolved since the minimum frequency is $\omega_{\min }=2 \pi / T$ and the maximum frequency is $\omega_{\max }=\pi / \Delta t^{\prime}$. In supplementary material, the video compares the fields $b, b^{w}, b^{e}$ for $N=70$.

\section{Balance of energy between waves and eddies}

\subsection{Energy of waves and eddies}

The total mechanical energy $E^{T}=E_{u}^{T}+E_{b}^{T}$ is the sum of kinetic energy $E_{u}^{T}$ and potential energy $E_{b}^{T}$. Based on our orthogonal decomposition, we split these energies into their wave and eddy parts as $E^{T}=E^{w}+E^{e}$ and $E^{l}=E_{u}^{l}+E_{b}^{l}$, with 
$E_{u}^{l}=0.5<\hat{\mathbf{u}}^{l}, \hat{\mathbf{u}}^{l}>$ and $E_{b}^{l}=0.5 N^{-2}<\hat{b}^{l}, \hat{b}^{l}>$ where $l$ stands for $w$ (wave), $e$ (eddy) or $T$ (total) and $<,>$ is defined in section 2 . The eddy part of the poloidal kinetic energy is defined as $E^{p, e}=0.5<\hat{\mathbf{u}}^{p, e}, \hat{\mathbf{u}}^{p, e}>$.

Figures $2 \mathrm{a}$ and $2 \mathrm{~b}$ show for both resolutions, the energy distribution between waves and eddies, $E^{e}$ and $E^{w}$ compared to total energy $E^{T}$. Since two parameters $F r$ and $R e_{b}$ appear to be strongly correlated (recall that $R e_{b}=R e_{h} F r^{2}$ ), we plot the distribution against $\mathrm{Fr}$ and $R e_{b}$ separately. Moreover, we can observe the evolution of these energies either at constant $R e_{b}$ and weakly increasing $\mathrm{Fr}$ (from high to low resolution on figure $2 \mathrm{a}$ ), or at constant $F r$ and weakly decreasing $R e_{b}$ (from high to low resolution on figure 2b). As expected, on figure 2 we observe that the eddy part of any form of energy $E^{e}$ increases and the wave part $E^{w}$ decreases, both when $R e_{b}$ increases (figure $2 \mathrm{a}$ ) and when $F r$ increases (figure $2 \mathrm{~b}$ ). Staquet \& Godeferd (1998) found a similar distribution of kinetic energy (60\% to eddies and $40 \%$ to waves) at $F r \simeq 0.006$ for decaying turbulence. Moreover, at fixed $R e_{b}$ (figure 2 2 ), when $F r$ increases, there is more energy in the eddy part than in the wave part, as expected by the meaning of $F r$ (inertial effects are more important than gravity effects). By increasing $F r$, the evolution of $E^{w}, E^{e}$ as a function of $R e_{b}$ seems to be shifted to smaller values of $R e_{b}$ as well as towards the equilibrium point where $E^{w}=E^{e}$. Nevertheless, at fixed $\operatorname{Fr}$ (figure $2 \mathrm{~b}$ ), when $R e_{b}$ decreases, there is more energy in the eddy part than in the wave part, which is not obvious. Once again, this evolution seems to be shifted towards a smaller value of $\mathrm{Fr}$.

To analyse this result, we must analyse the composition of each type of energy. Figures $2 \mathrm{a}$ and $2 \mathrm{~b}$ show the ratio of potential and kinetic energy distribution of waves $\left(E_{b}^{w}, E_{u}^{w}\right)$ and eddies $\left(E_{b}^{e}, E_{u}^{e}\right)$ compared to total energy $E^{T}$. First, we observe that the buoyancy energy $E_{b}^{w}$ and kinetic energy $E_{u}^{w}$ contain the same percentage of total energy of waves, i.e. $E_{b}^{w} \sim E_{u}^{w}$ for any $R e_{b}$ or any $F r$, as generally expected for gravity waves. Secondly, we observe that the buoyancy energy of eddies is less than kinetic energy of eddies i.e. $E_{u}^{e}>E_{b}^{e}$ for any $F r$ or $R e_{b}$. Nevertheless, at fixed $\mathrm{Fr}$, for instance at $\mathrm{Fr} \sim 0.014$, when $R e_{b}$ decreases there is more kinetic energy in eddy for lower $R e_{b}$ i.e. $E_{u}^{e}\left(F r=0.014, R e_{b}=\right.$ $0.7)>E_{u}^{e}\left(F r=0.013, \operatorname{Re}_{b}=1.8\right)$.

This non obvious result can be analysed by decomposing the kinetic energy of eddies into poloidal and toroidal parts. By following the decomposition (2.7) and (2.8), the eddy part can be decomposed into poloidal and toroidal components: $E_{u}^{e}=E_{u}^{p, e}+E_{u}^{t, e}$ where $E_{u}^{p, e}=0.5<\hat{\mathbf{u}}^{e} \cdot \mathbf{e}^{\mathbf{p}}, \hat{\mathbf{u}}^{e} \cdot \mathbf{e}^{\mathbf{p}}>$ and $E_{u}^{t, e}=0.5<\hat{\mathbf{u}}^{e} \cdot \mathbf{e}^{\mathbf{t}}, \hat{\mathbf{u}}^{e}$. $\mathbf{e}^{\mathrm{t}}>$. On figures $2 \mathrm{a}$ and $2 \mathrm{~b}$ we have only plotted $E_{u}^{p, e}$, from which the value of $E_{u}^{t, e}=E_{u}^{e}-E_{u}^{p, e}$ can be deduced. We observe the same percentage of total energy in the poloidal and buoyancy eddy energy, i.e. $E_{b}^{e} \sim E_{u}^{p, e}$ independently of $\mathrm{Fr}$ or $R e_{b}$. This percentage increases slowly with $R e_{b}$ or with $F r$. Note that total potential energy $E_{b}^{T}=E_{b}^{w}+E_{b}^{e}$ is linked to the available potential energy (see section 14.1 in Davidson (2013)). The available potential energy is a mechanical form of gravitational potential energy that stores the energy of an unstable density pattern i.e. the light- and heavy-density fluid parcels are not in equilibrium. While a part of this unstable configuration is related to the IGW $\left(E_{b}^{w}\right)$ as the waves induce spatial variations of density fluctuation, the other part $\left(E_{b}^{e}\right)$ contains, among other things, the density overturns (light density over heavy density). It seems that the equality $E_{b}^{e} \sim E_{u}^{p, e}$ reflects the effect of eddies in the vertical plane, which is directly related to the poloidal part of the velocity field. Since $E_{b}^{e} \sim E_{u}^{p, e}$ is more or less constant with $R e_{b}$ at fixed $F r$, this means that only the toroidal 
part $E_{u}^{t, e}$ increases when $R e_{b}$ decreases. The increase of $E_{u}^{t, e}$ leads to an increase of the total kinetic energy of the eddy part $E_{u}^{e}$. We remind that the toroidal part $\left(k_{h} \neq 0\right)$ does not include the $\operatorname{VSHF}\left(k_{h}=0\right)$. Generally, large scales contain more kinetic energy than small scales dominated by the dissipation, so that the large scale vortical modes are well represented by the toroidal part of energy. We therefore argue that the relative increase - with respect to total energy - of the part of kinetic energy in the vortical modes can be associated with an increase in large, smooth and stable horizontal layers as the flow is more and more in the VASF regime by decreasing $R e_{b}$, as in the nomenclature by Brethouwer et al. (2007). This observation could explain the shift of the distribution of energy $E^{w}$, $E^{e}$ towards smaller $F r$ as $R e_{b}$ decreases.

Note that at very large $R e_{b}$, it seems that $E_{u}^{p, e} \sim E_{b}^{e} \sim E_{b}^{w} \sim E_{u}^{w}$. In the original decomposition by Riley, at large $R e_{b}$ all the potential energy of eddies $E_{b}^{e}$ and the poloidal part of kinetic energy of eddies $E_{u}^{p, e}$ are wrongly assigned to the wave part (i.e. $\sim 10 \%$ for each part), thus inducing a departure of $40 \%$ in comparison to our results: $+20 \%$ of energy in IGW and $-20 \%$ of energy in eddies.

\subsection{Flux of waves and eddies}

The evolution of total energy in stratified turbulence is driven by the flux of energy in equation $d E^{T} / d t=P-\varepsilon_{T}$ where the total dissipation $\varepsilon_{T}=\varepsilon_{u}+\varepsilon_{b}$ with $\varepsilon_{u}=\nu<k^{2} \hat{\mathbf{u}}, \hat{\mathbf{u}}>$ and $\varepsilon_{b}=\mathcal{X} N^{-2}<k^{2} \hat{b}, \hat{b}>$. During the statistically stationary regime, the total energy stored is constant, so that $d E^{T} / d t=0$ and, for all stratification intensities, the output flux balances the input flux as $P \simeq \varepsilon_{T}$. The wave and eddy decomposition now permits to address the question about how do wave- and eddy-related fluxes evolve with stratification?

We consider the equation of evolution of wave and eddy parts described by Verma (2019) as Lin-type equations, and hence that for total energy. We start

with equations $\widehat{(2.1)}$ and $\widehat{(2.2)}$ as space Fourier-transformed equations $(2.1)$ and (2.2). We also use the space Fourier-transformed decomposition of velocity and density $(2.8)$, and we project both equations $\sqrt{2.17}$ and $\sqrt{2.2}$ on the wave part using the inner products $\left[\widehat{(2.1)}, \hat{\mathbf{u}}^{w}(\mathbf{k}, t)\right]$ and $\left[\widehat{(2.2)}, \hat{b}^{w}(\mathbf{k}, t)\right]$, and on the eddy part using $\left[\widehat{[2.1]}, \hat{\mathbf{u}}^{e}(\mathbf{k}, t)\right]$, and $\left[\widehat{[2.2}, \hat{b}^{e}(\mathbf{k}, t)\right]$, relying on orthogonality properties noted in section 2. This yields four $(\mathbf{k}, t)$-dependent equations for potential/kinetic energy and for the wave/eddy part. Balance equations are finally obtained by summing the potential and kinetic energies and using the spectral integration $<,>$ :

$$
\begin{aligned}
& d E^{w} / d t=T_{e e}^{w}+T_{w e}^{w}-\varepsilon_{T}^{w}+P^{w} \\
& d E^{e} / d t=T_{w w}^{e}+T_{e w}^{e}-\varepsilon_{T}^{e}+P^{e}
\end{aligned}
$$

where $\varepsilon_{T}^{l}=\varepsilon_{u}^{l}+\varepsilon_{b}^{l}$ with $\varepsilon_{u}^{l}=\nu<k^{2} \hat{\mathbf{u}}^{l}, \hat{\mathbf{u}}^{l}>$ and $\varepsilon_{b}^{l}=\mathcal{X} N^{-2}<k^{2} \hat{b}^{l}, \hat{b}^{l}>$ is the total dissipation rate for each part $l=w, e$, the exchange term $T_{i j}^{l}=<$ $\widehat{\mathbf{u}^{i} \times \boldsymbol{\omega}^{j}}, \hat{\mathbf{u}}^{l}>-N^{-2}<\widehat{\mathbf{u}^{i \cdot \nabla} b^{j}}, \hat{b}^{l}>$ and the injected power $P=P^{w}+P^{e}=10$ for each part $P^{l}=<\hat{\mathbf{F}}_{\mathbf{u}}, \hat{\mathbf{u}}^{l}>$. In equations 4.1 , , one neglects the interactions $T_{s j}^{l}$ of waves and eddies with VSHF because in our simulations these terms are small compared to others (for $N=100, T_{s j}^{l} \sim \mathcal{O}\left(10^{-7} T_{i j}^{l}\right)$ ). As discussed by Verma (2019), triadic transfers are such that $T_{i j}^{l}=-T_{i l}^{j}$ so that $T_{w e}^{w}=-T_{w w}^{e}$ 

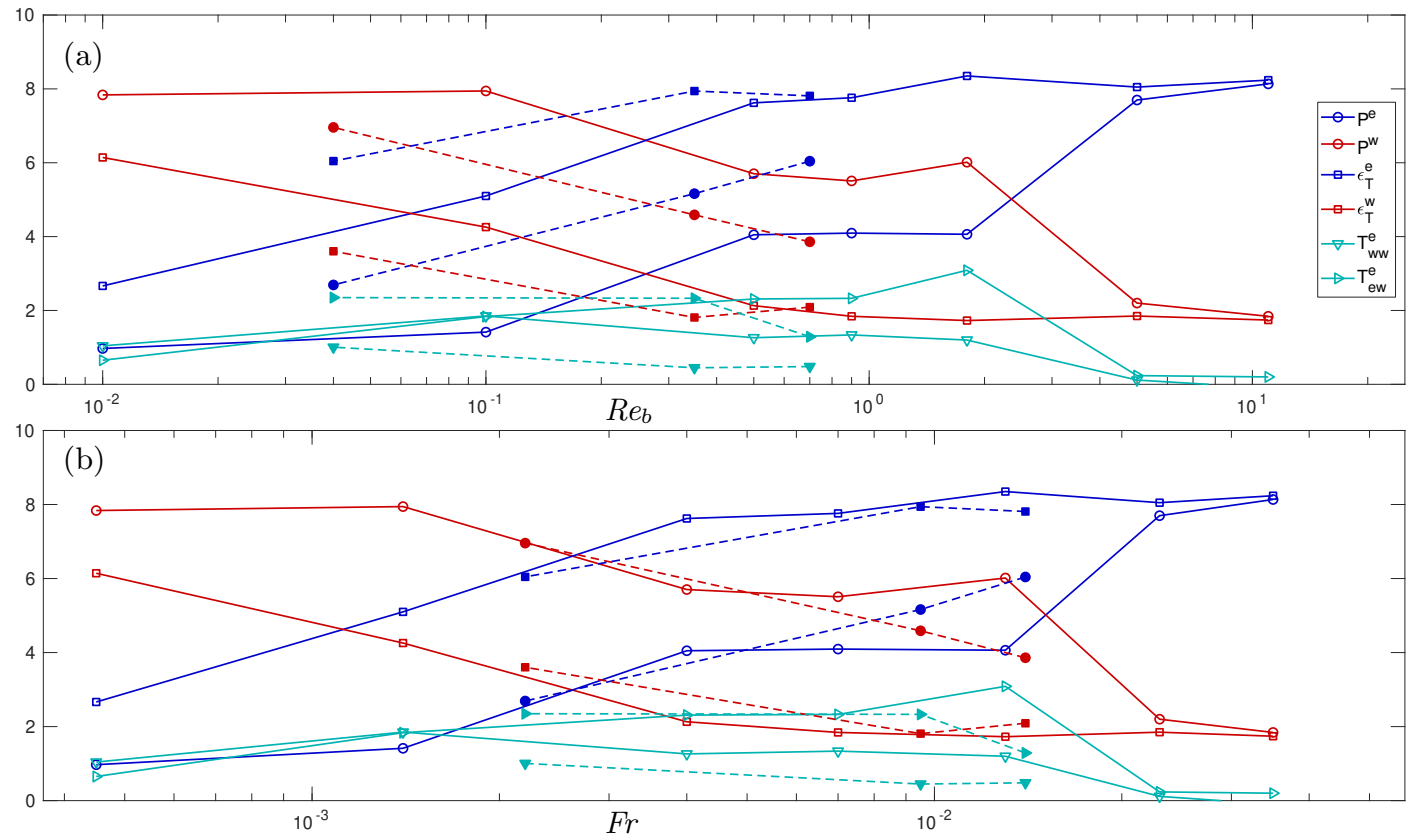

Figure 3: Evolution with (a) $R e_{b}$, and (b) Fr, of the contributions of forcing $P^{l}$, dissipation $\varepsilon^{l}$ and transfer $T_{i w}^{e}$ from waves to eddies. The transfer from eddies to waves is easily computed as the inverse of the transfer from waves to eddies

$T_{i e}^{w}=-T_{i w}^{e}$. Numerical simulations with $512^{3}$ points correspond to open symbols and solid lines, numerical simulations with $256^{3}$ points to filled symbols and dotted lines.

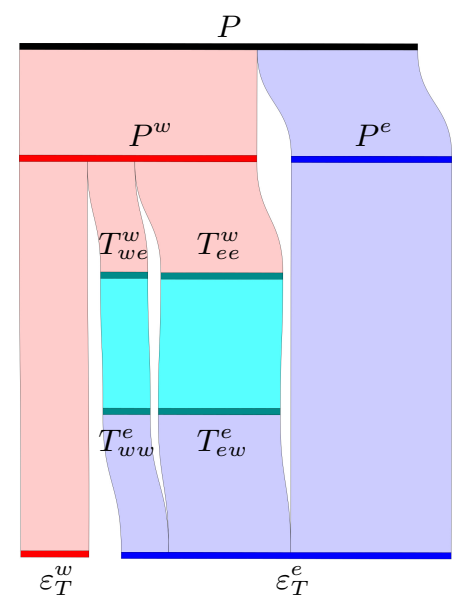

Figure 4: Sankey's diagram of energy flux at $R e_{b}=1.8$ and $F r=0.013$ (see text).

and $T_{e w}^{e}=-T_{e e}^{w}$. $T_{i j}^{l}$ is an energy exchange term between $l$ and $j$ parts, due to the interaction between the part $j$ 'convected' by part $i$ that exchanges energy with part $l$. Thus, $T_{i j}^{j}=0$ so that such terms are not net exchange terms, but are dynamically similar to convection terms, since they convey the modification of part $j$ by part $i$ that acts onto part $j$. For instance, $T_{e e}^{e}=0$ and $T_{w w}^{w}=0$ are respectively similar to a classical non-linear transfer between eddies and to a non- 
linear transfer between waves. In the statistically stationary regime, $d E^{w, e} / d t=0$ and the equilibrium of the fluxes is reached since all ters compensate one another.

Figures $3 \mathrm{a}$ and $3 \mathrm{~b}$ show, for both resolutions, the evolution with $R e_{b}$ and with $\mathrm{Fr}$ of the amount of the different terms in equation (4.1). Again, we can estimate the evolution of these values either at fixed $R e_{b}$ and weakly increasing $\mathrm{Fr}$ (from high to low resolution) or at fixed $\mathrm{Fr}$ and weakly decreasing $R e_{b}$ (from high to low resolution). In order to facilitate physical interpretation, we show a corresponding flux diagram (a.k.a. Sankey's diagram) in figure 4 for $R e_{b}=1.8$ and $F r=0.013$ to visualize quantitatively the energy flux from the injection $P$ to the two dissipations $\varepsilon_{T}^{w}$ and $\varepsilon_{T}^{e}$, either directly, or indirectly by wave/eddy exchange terms $T_{i j}^{l}$. Each band represents a component of the balance of energy, with a width proportional to the energy flux it involves. Red, blue and cyan respectively indicate the wave, eddy and exchange parts.

Figures 3 and 3 b show that at $\operatorname{Re}_{b}>\operatorname{Re}_{b}^{T} \simeq 2$ and $F r>F r^{T} \simeq 0.02$, the input power for waves and eddies is in balance exclusively with the dissipation, i.e. $P^{e} \simeq \varepsilon_{T}^{e}$ and $P^{w} \simeq \varepsilon_{T}^{w}$ and there is no exchange between waves and eddies. This does not mean that there is no transfer between waves and eddies, it only means that, in overall, no net transfer occurs, but a scale-by-scale transfer (a 'cascade') is still possible between them. Moreover, injected energy is mainly pumped by eddies since $P^{e}>P^{w}$. This changes completely when $R e_{b}$ decreases or $\mathrm{Fr}$ decreases as $\operatorname{Re}_{b}<R e_{b}^{T}$ or $F r<F r^{T}$. Indeed, in the most stratified case $F r=0.00045$ at low $R e_{b}=0.01$, the input power and dissipation are more important for the wave part than for the eddy part $\left(P^{w}>P^{e}\right.$ and $\left.\varepsilon_{T}^{w}>\varepsilon_{T}^{e}\right)$ and the exchange terms $T_{w e}^{w}$ and $T_{e e}^{w}$ remove energy from waves $\left(P^{w}>\varepsilon_{T}^{w}\right)$ and redistribute it to eddies $\left(P^{e}<\varepsilon_{T}^{e}\right)$. As $R e_{b}$ increases and $F r$ increases close to the transition $R e_{b} \sim R e_{b}^{T}$ and $F r=F r^{T}$, the dissipation associated with eddies gets larger $\left(\varepsilon_{T}^{e}>\varepsilon_{T}^{w}\right)$ as expected but, surprisingly, the input power for waves remains large and there is a significant transfer from the wave part to the eddy part which amounts to a total up to $50 \%$ of the eddy dissipation. During this transition, the exchange between wave and eddy is dominant. Similarly, in Godeferd \& Cambon (1994), a lot of the energy appears to be pumped from the waves by the exchange term $T_{w e}^{w}$. In this transition zone, at fixed $R e_{b}$, when $F r$ increases, as expected, the eddy part takes more importance and the evolution seems to be shifted to a lower $R e_{b}^{T}$. Nevertheless, by comparing the numerical simulations with $512^{3}$ points and $256^{3}$ points, it appears that the transfer mostly depends on the Froude number, although its amplitude varies slightly between the two resolutions. Moreover, the way the forcing and the dissipation is distributed between waves and eddies seems relatively invariant against the Froude number.

These observations result in a global analysis of transfers between waves and eddies: the global exchange is zero for the exchange terms, i.e. $T_{e e}^{e}=T_{w w}^{w}=T_{e w}^{w}=$ $T_{w e}^{e}=0$, but these terms are associated to 'cascades' and therefore influence indirectly the transfers between wave and eddies. For example, the global term $T_{e w}^{w}=0$, meaning there is no global exchange, but there is still a scale-by-scale transfer between waves aided by an eddy that acts as a mediator (Verma 2019).

\section{Mixing by waves or eddies}

The above wave/eddy flow decomposition also permits to understand the contribution of IGW and eddies to mixing. The total mixing coefficient is defined by $\Gamma=\varepsilon_{b} / \varepsilon_{u}$ (Peltier \& Caulfield 2003). For oceanographic application, the 

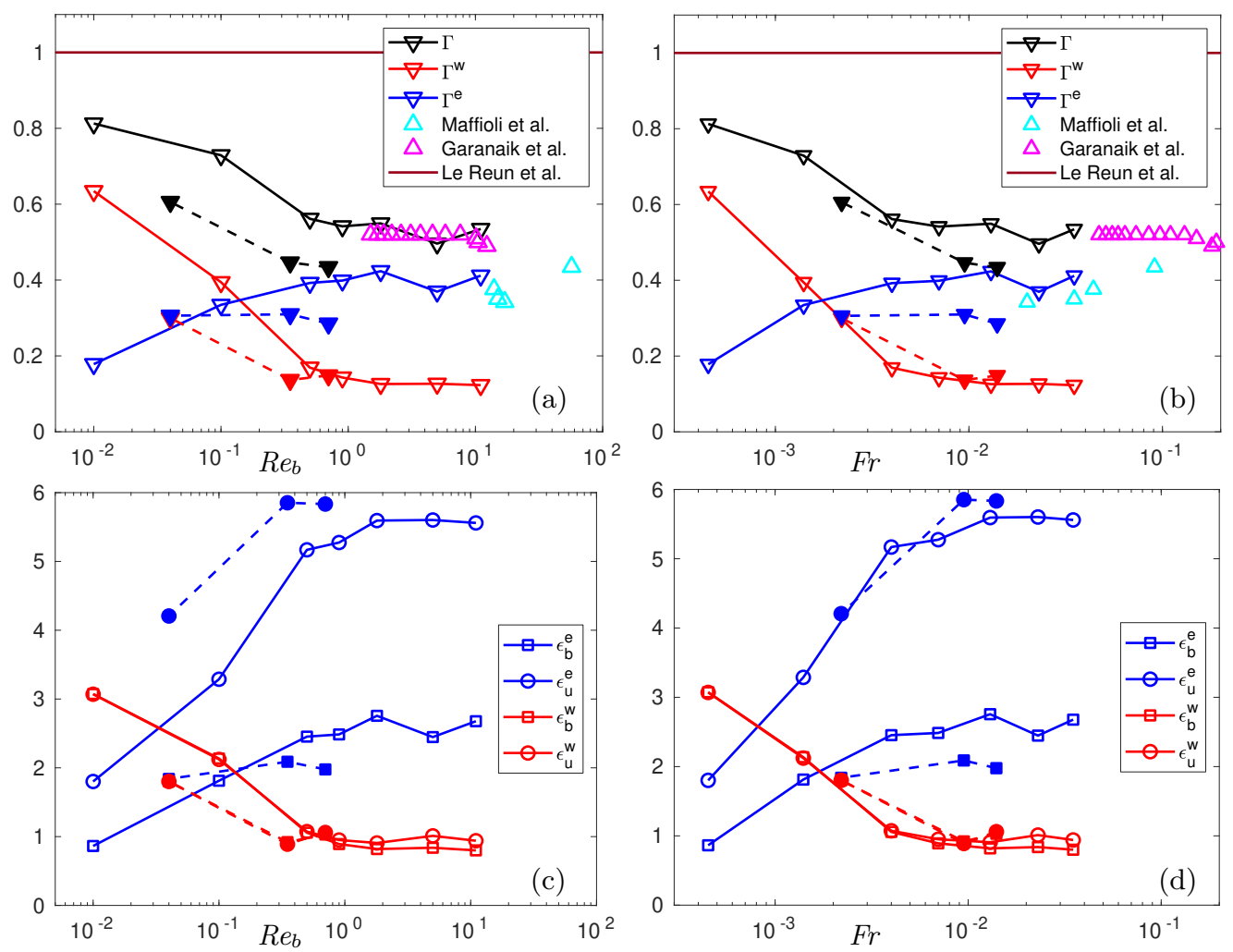

Figure 5: Mixing coefficients $\Gamma, \Gamma^{w}, \Gamma^{e}$ compared with data from literature against (a) $R e_{b}$, and (b) Fr. Kinetic and buoyancy dissipation for waves and eddies against (c) $R e_{b}$, and (d) Fr. Numerical simulations with $512^{3}$ points are shown by open symbols and solid lines, and numerical simulations with $256^{3}$ points with filled symbols and dotted lines.

eddy diffusity of density $\kappa_{\rho}$ can be used for parameterizing the stratification mixing with equation $\kappa_{\rho}=\mathcal{X} \operatorname{Pr} \Gamma \operatorname{Re}_{b}$ (Caulfield 2021). In this formulation, it is possible that a highly efficient mixing at low $R e_{b}$ lead to a smaller eddy diffusity of density than flows with a higher $R e_{b} . \Gamma$ is also useful for calculating the vertical diffusivity of density used in the model proposed by Osborn (1980). Whereas $\Gamma$ was approximated to a constant $\Gamma \simeq 0.2$ in the ocean where $R e_{b} \sim 100-1000$ (Mashayek et al. 2017), recent DNS in decaying stratified turbulence at resolution $512^{3}$ (Garanaik \& Venayagamoorthy 2019) and forced stratified DNS at larger resolution (Maffioli et al. 2016), suggest a dependence of $\Gamma$ with $F r$ and $R e_{b}$. On figure 5b, we have reported these authors' values for $\Gamma$ in a Froude range similar to ours, i.e. $F r \ll 1$ and associated with $R e_{b} \simeq 10-20$ (forced case) and $R e_{b} \simeq 1-10$ (decaying case). Moreover, in a wave regime of superposed low-amplitude IGW with weak nonlinear interactions, Le Reun et al. (2018) find that $\Gamma=1 / \operatorname{Pr}=1$.

Our simulations explore the transition between these two regimes. In order to understand separately the effect of waves and eddies on mixing, we therefore separate the total mixing coefficient $\Gamma=\varepsilon_{b} / \varepsilon_{u}=\Gamma^{w}+\Gamma^{e}$ into mixing due to waves $\Gamma^{w}=\varepsilon_{b}^{w} /\left(\varepsilon_{u}^{e}+\varepsilon_{u}^{w}\right)$ and mixing due to eddies $\Gamma^{e}=\varepsilon_{b}^{e} /\left(\varepsilon_{u}^{e}+\varepsilon_{u}^{w}\right)$ by using $\varepsilon_{u}=\varepsilon_{u}^{e}+\varepsilon_{u}^{w}$ and $\varepsilon_{b}=\varepsilon_{b}^{e}+\varepsilon_{b}^{w}$. On figures $5 \mathrm{a}$ and $5 \mathrm{~b}$, the coefficients $\Gamma$, 
$\Gamma^{w}, \Gamma^{e}$ are plotted versus $R e_{b}$ and $F r$ respectively, and compared to the abovementioned data. Our coefficient values seem to coincide better with a variation in Fr rather than with a variation in $R e_{b}$ (as in the flux analysis discussed previously in section 4.2). For $R e_{b} \geqslant 1$ and $F r \geqslant 10^{-2}$, we find a value $\Gamma \simeq 0.5$ similar to that in Garanaik \& Venayagamoorthy (2019) at similar resolution and slightly lower Froude number. Moreover, we observe that the wave mixing and eddy mixing reach a plateau, as expected, but the eddies mix more than waves since $\Gamma^{e} \simeq 0.4 \geqslant \Gamma^{w} \simeq 0.1$. Note that our decomposition considers as eddies, among others, the breaking of internal waves or overturning with vertical velocity. This could nuance the belief that overturning is the main source of mixing (Gregg et al. 2003). The global mixing coefficient found by Maffioli et al. (2016) is close to our mixing coefficient by eddies $\Gamma^{e}$. As their DNS are done at a higher $R e_{b} \simeq 10-20$, it is possible that their flows contain mostly eddies, resulting in a mixing coefficient dependent only on mixing by eddies. When $R e_{b} \rightarrow 0$, the total mixing increases and tends to $\Gamma \simeq 1$ as expected by Le Reun et al. $(2018)$. In this case, $\Gamma^{w}$ increases a lot, whereas $\Gamma^{e}$ decreases. Indeed, we expect at very low buoyancy Reynolds number that the waves dominate the flow and become the main factor of mixing. At fixed $F r$, when $R e_{b}$ decreases, the same physics is shifted to low $R e_{b}$ but in a non-obvious way the plateau value seems to be constant for wave mixing $\Gamma^{w} \simeq 0.1$, while mixing by eddy seems to be weaker $\Gamma^{e} \simeq 0.3$.

In order to better understand the physical phenomena underlying mixing and its modelling, an in-depth analysis of the different dissipation terms is necessary. Note that while kinetic and buoyancy energies are more related to large scales, the different dissipations are related to small scales. On Figures $5 \mathrm{c}$ and $5 \mathrm{~d}$, we have plotted the different contributions to dissipation as functions of $R e_{b}$ and $F r$. Note that during statistically stationary regime, the constant forcing $P=10$ and dissipation are in balance so that $P \simeq \varepsilon_{u}^{e}+\varepsilon_{u}^{w}+\varepsilon_{b}^{e}+\varepsilon_{b}^{w}$. First, as expected for IGW, the kinetic and potential dissipations of the waves are equal, i.e. $\varepsilon_{b}^{w} \sim \varepsilon_{u}^{w}$. Moreover, this confirms the idea proposed by Le Reun et al. (2018) that $\Gamma=1$ is always true for the IGW even if they are mixed with eddies. Secondly, the kinetic dissipation of eddies is greater than the potential dissipation of eddies i.e. $\varepsilon_{u}^{e}>\varepsilon_{b}^{e}$. Thirdly, all these values tend towards a plateau when $F r$ is large and $R e_{b} \simeq 1$, with $\varepsilon_{b}^{w} \sim \varepsilon_{u}^{w} \sim \varepsilon_{u}^{e} / 6$. In this regime, as all the statistics $\epsilon_{b}^{w, e}$ and $\epsilon_{u}^{w, e}$ involved reach a plateau, so does the mixing coefficient. Apparently, our coefficient values seem to coincide better with a variation in $F r$ rather than a variation in $R e_{b}$ which shifts the evolution to a lower $R e_{b}$. At fixed $F r$ and decreasing $R e_{b}$, all dissipation terms remain unchanged except $\varepsilon_{b}^{e}$, which implies that the mixing $\Gamma^{e}$ due to eddies decreases.

\section{Conclusion and Perspectives}

We have developed and used an extension of Riley's decomposition (Riley et al. 1981) to separate waves and eddies in a stratified flow. This method takes into account the vertical mixing and the temporal/spatial properties of waves with their modifications by the mean flow (VSHF). We apply this analysis on DNS results with strong stratification $(F r \leqslant 1)$, but varying buoyancy Reynolds number over a wide range of regimes, from a viscously affected stratified regime $\left(R e_{b} \ll 1\right)$ to stably stratified turbulence regime $\left(R e_{b} \gg 1\right)$. From this separation, as expected, we show that the total wave energy dominates for $R e_{b} \ll 1$ and the total eddy energy dominates for $R e_{b} \gg 1$. There is an equipartition in the 
wave energy between the kinetic and the potential part which indicates that we succeeded in obtaining a good extraction of the IGW in the turbulent mixing for any $R e_{b}$. Moreover, at large $R e_{b}$, the potential energy of eddies is close to the potential or kinetic energy of waves.

We establish the balance equations of energies separately for wave and eddy parts. In a statistically stationary regime, the different fluxes of the energy balance are studied from input forcing to output dissipation and by taking into account the exchange between parts. We observe that dissipation by eddies is more efficient than dissipation by waves except at largest stratification. This higher efficiency comes from an exchange that pumps the energy from the wave part and sends it to the eddy part, where it is dissipated. This scenario seems valid from low turbulence at $R e_{b} \ll 1$ to higher turbulence at $R e_{b} \sim 1$. In addition, the dissipation of kinetic energy and potential energy have the same values, again showing that the characteristics of IGW at small scales are also adequately captured by our method. For $R e_{b} \gg 1$, the input power is directly dissipated by each part separately, with no global exchange. However, the study of transfer by waves and eddies with themselves will require further scale-by-scale analysis in order to identify their specific contributions to the turbulent cascade, and gain a better understanding of the internal mechanisms of energy transfer and cascade. However this is outside of the scope of this article. In addition, our present analysis includes a forcing that disregards the energy partition between waves and eddies. As a result, the dissipation terms are close to the energy input of waves and eddies. Another study with wave- and eddy-specific forcing is required to address this.

Our analysis also provides access to dissipation, and therefore to the contributions of waves and eddies to the mixing coefficient $\Gamma$. A result particularly relevant for mixing models is the plateau reached at high buoyancy Reynolds number $R e_{b}$ by split mixing contributions, showing that eddy mixing is four times that of waves for our higher resolution. However, it would be relevant to explore higher resolution results in order to obtain $\Gamma^{w}$ and $\Gamma^{e}$ values at a much higher $R e_{b}$ while keeping $\mathrm{Fr}$ small. We assumed $\operatorname{Pr}=1$ in our study. Nevertheless, (Smyth et al. 2001) show that the Prandtl number can have a large effect on mixing, especially important when considering ocean mixing. Moreover, as remarked above for fluxes, the use of constant power forcing discriminated between the wave and eddy parts could further advance our knowledge of the direct impact of wave-eddy interaction on mixing.

Funding. This research was funded by ANR DisET grant number ANR-17-CE30-0003. This work was granted access to the HPC resources of IDRIS under the allocation A0062A02206 made by GENCI and HPC ressources of the FLMSN, partner of EQUIPEX EQUIP@MESO.

Declaration of interests. The authors report no conflict of interest.

Author ORCID. H. Lam, https://orcid.org/0000-0002-2604-2714;

F. S. Godeferd, https://orcid.org/0000-0002-8898-5451

\section{REFERENCES}

Bartello, P. 1995 Geostrophic adjustment and inverse cascades in rotating stratified turbulence. J. Atmos. Sci. 52 (24), 4410-4428.

Brethouwer, G., Billant, P., Lindborg, E. \& Chomaz, J.-M. 2007 Scaling analysis and simulation of strongly stratified turbulent flows. J. Fluid Mech. 585, 343-368. 
CAUlfield, C.P. 2021 Layering, instabilities, and mixing in turbulent stratified flows. Annual Review of Fluid Mechanics 53 (1), 113-145.

Cusack, J. M., Brearley, J. A., Naveira Garabato, A. C., Smeed, D. A., Polzin, K. L., Velzeboer, N. \& Shakespeare, C. J. 2020 Observed Eddy-Internal Wave Interactions in the Southern Ocean. J. Phys. Ocean. 50 (10), 3043-3062.

Davidson, Peter Alan 2013 Turbulence in rotating, stratified and electrically conducting fluids. Cambridge University Press.

Di Leoni, P. C., Cobelli, P. J. \& Mininni, P. D. 2015 The spatio-temporal spectrum of turbulent flows. The European Physical Journal E 38 (136).

Di Leoni, P. C. \& Mininni, P. D. 2015 Absorption of waves by large-scale winds in stratified turbulence. Phys. Rev. E 91 (3), 033015.

Garanaik, A. \& Venayagamoorthy, S. K. 2019 On the inference of the state of turbulence and mixing efficiency in stably stratified flows. J. Fluid. Mech. 867, 323-333.

Godeferd, F. S. \& CAmbon, C. 1994 Detailed investigation of energy transfers in homogeneous stratified turbulence. Phys. Fluids 6 (6), 2084-2100.

GregG, M. C., Sanford, T. B. \& Winkel, D. P. 2003 Reduced mixing from the breaking of internal waves in equatorial waters. Nature 422 (6931), 513-515.

Herbert, C., Marino, R., Rosenberg, D. \& Pouquet, A. 2016 Waves and vortices in the inverse cascade regime of stratified turbulence with or without rotation. J. Fluid. Mech. 806, 165-204.

Ivey, G.N., Winters, K.B. \& KosefF, J.R. 2008 Density stratification, turbulence, but how much mixing? Annual Review of Fluid Mechanics 40 (1), 169-184, arXiv: https://doi.org/10.1146/annurev.fluid.39.050905.110314.

Kimura, Y. \& Herring, J. R. 2012 Energy spectra of stably stratified turbulence. J. Fluid. Mech. 698, 19-50.

Lam, H., Delache, A. \& Godeferd, F. S. 2020 Partitioning Waves and Eddies in Stably Stratified Turbulence. Atmosphere 11 (4), 420.

Le Reun, T., Favier, B., Barker, A. J. \& Le Bars, M. 2017 Inertial wave turbulence driven by elliptical instability. Phys. Rev. Let. 119 (3).

Le Reun, T., Favier, B. \& Le Bars, M. 2018 Parametric instability and wave turbulence driven by tidal excitation of internal waves. J. Fluid. Mech. 840, 498-529.

LELONG, M.-P. \& RiLEY, J. J. 1991 Internal wave-vortical mode interactions in strongly stratified flows. J. Fluid. Mech. 232, 1-19.

Lindborg, E. \& Brethouwer, G. 2007 Stratified turbulence forced in rotational and divergent modes. J. Fluid. Mech. 586, 83.

Lvov, Yuri V, Polzin, Kurt L, Tabak, Esteban G \& Yokoyama, Naoto 2010 Oceanic internal-wave field: theory of scale-invariant spectra. J. Phys. Ocean. 40 (12), 2605-2623.

Mackinnon, J. A. ET AL. 2017 Climate Process Team on Internal Wave-Driven Ocean Mixing. Bull. Am. Meteo. Soc. 98 (11), 2429-2454.

Maffioli, A., Brethouwer, G. \& Lindborg, E. 2016 Mixing efficiency in stratified turbulence. J. Fluid. Mech. 794, R3.

Maffioli, Andrea, Davidson, PA, Dalziel, SB \& Swaminathan, Nedunchezhian 2014 The evolution of a stratified turbulent cloud. Journal of fluid mechanics 739, 229.

Maffioli, A., Delache, A. \& Godeferd, F. S. 2020 Signature and energetics of internal gravity waves in stratified turbulence. Phys. Rev. Fluids 5, 114802.

Mashayek, A., Salehipour, H., Bouffard, D., Caulfield, C. P., Ferrari, R., Nikurashin, M., Peltier, W. R. \& Smyth, W. D. 2017 Efficiency of turbulent mixing in the abyssal ocean circulation. Geophys. Res. Let. 44 (12), 6296-6306.

Moulin, F. \& FlóR, J.-B. 2006 Vortex-wave interaction in a rotating stratified fluid: Wkb simulations. J. Fluid. Mech. 563, 199-222.

MÜLleR, P. 1976 On the diffusion of momentum and mass by internal gravity waves. J. Fluid. Mech. 77 (4), 789-823.

Müller, Peter, Holloway, Greg, Henyey, Frank \& Pomphrey, Neil 1986 Nonlinear interactions among internal gravity waves. Rev. Geophys. 24 (3), 493-536.

OlBERs, DiRK J 1976 Nonlinear energy transfer and the energy balance of the internal wave field in the deep ocean. J. Fluid. Mech. 74 (2), 375-399. 
Osborn, T. R. 1980 Estimates of the Local Rate of Vertical Diffusion from Dissipation Measurements. J. Phys. Oceano. 10 (1), 83-89.

Peltier, W. R. \& Caulfield, C. P. 2003 Mixing efficiency in stratified shear flows. Ann. Rev. Fluid Mech. 35 (1), 135-167.

Riley, J. J., Metcalfe, R. W. \& Weissman, M. A. 1981 Direct numerical simulations of homogeneous turbulence in density-stratified fluids 76 (1), 79-112.

Savaro, C., Campagne, A., Linares, M. C., Augier, P., Sommeria, J., Valran, T., Viboud, S. \& Mordant, N. 2020 Generation of weakly nonlinear turbulence of internal gravity waves in the Coriolis facility. Phys. Rev. Fluids 5, 073801.

Smith, Leslie M \& Waleffe, Fabian 1999 Transfer of energy to two-dimensional large scales in forced, rotating three-dimensional turbulence. Physics of fluids 11 (6), 1608-1622.

Smith, L. M. \& Waleffe, F. 2002 Generation of slow large scales in forced rotating stratified turbulence. J. Fluid. Mech. 451, 145-168.

Smyth, W. D., Moum, J. N. \& Caldwell, D. R. 2001 The efficiency of mixing in turbulent patches: Inferences from direct simulations and microstructure observations. Journal of Physical Oceanography 31 (8), 1969 - 1992.

Staquet, C. \& Godeferd, F. S. 1998 Statistical modelling and DNS of decaying stably stratified turbulence. Part 1. flow energetics. J. Fluid. Mech. 360, 295-340.

Verma, M. K. 2019 Energy Transfers in Fluid Flows: Multiscale and Spectral Perspectives. Cambridge University Press.

Waite, Michael L \& Bartello, Peter 2004 Stratified turbulence dominated by vortical motion. Journal of Fluid Mechanics 517, 281. 